\title{
Changes in Cerebral Blood Flow and Prolonged Disturbances in Cerebral Autoregulation in Critically ill Children with Diabetic Ketoacidosis
}

\author{
Melissa G Chung ${ }^{* 1,2}$, Tensing $\mathrm{Maa}^{1}$ and Nicole F O'Brien ${ }^{1}$ \\ ${ }^{1 *}$ Department of Pediatrics, Nationwide Children's Hospital, The Ohio State University, Ohio \\ ${ }^{2}$ Division of Critical Care Medicine and Section of Neurology, Ohio \\ ${ }^{\star}$ Correspondence to: Melissa Chung, Section of Neurology, 700 Children's Drive, Columbus, Ohio 43205, Phone: 614-722-4625; \\ E-mail: Melissa.chung@nationwidechildrens.org
}

Received: December 03, 2016; Accepted: December 20, 2016; Published: January 18, 2017;

\begin{abstract}
Objective: Intracerebral complications are the most common cause of morbidity and mortality in children with diabetic ketoacidosis (DKA). Emerging evidence suggests that ischemic injury may be a factor in the development of cerebral edema. This study was designed to evaluate cerebral hemodynamics and autoregulation during DKA in critically ill children using transcranial Doppler ultrasound (TCD).
\end{abstract}

Design: Prospective observational study

Setting: Tertiary care pediatric intensive care unit (PICU)

Patients: Patients $\leq 18$ years admitted to the PICU with moderate to severe DKA (serum glucose $>200 \mathrm{mg} / \mathrm{dl}, \mathrm{pH}<7.15$, bicarbonate $<15 \mathrm{mmol} / \mathrm{L}$, and urine ketones).

Measurements and Main Results: Within 4 hours of admission the flow velocities in the middle cerebral arteries (MCA) and basilar artery (BA) were measured using TCD. Cerebral autoregulation was evaluated using the transient hyperemic response ratio (THRR), with values $\geq 1.1$ defined as normal. TCD was repeated after resolution of DKA. 26 patients were studied [median age $10 \mathrm{yrs}(0.75-18)$, median initial glucose 558 (289-1018) g/dL, median initial pH 6.98 (6.78-7.13)]. Mean MCA flow velocities were unchanged when compared to previously published normal values. Mean BA flow velocities were significantly lower than these normal values during and after resolution of DKA, $\mathrm{p}=0.001$. Cerebral autoregulation during DKA was impaired in $92 \%$ of patients [median THRR on right 0.97 (0.65-1.14), left 0.98 (0.39-1.26)] and remained abnormal in 64\% after resolution of DKA. Patients with clinical and imaging evidence of cerebral edema had lower BA mean flow velocities than patients without cerebral edema $(p=0.06)$.

Conclusions: Our data shows decreased basilar artery flow velocities and prolonged impairment of cerebral autoregulation in children with DKA. Understanding these disruptions in cerebral blood flow may lead to future therapeutic targets and should be further studied in hopes of improving neurologic outcomes in diabetic children experiencing DKA.

Keywords: diabetic ketoacidosis, blood flow velocity, cerebral blood flow, transcranial Doppler ultrasonography, cerebral edema, diabetes complications.

\section{Introduction}

Cerebral edema and intracerebral complications are the most common cause of morbidity and mortality in children with diabetic ketoacidosis (DKA) [1-4]. While symptomatic cerebral edema has been reported in approximately $1 \%$ of children with DKA, recent literature suggests that subclinical cerebral edema during DKA may be a more common occurrence than previously appreciated and may occur in up to $50 \%$ of children [1-8]. In one magnetic resonance imaging (MRI) study of children with DKA, 54\% of children had decreased ventricular size, which suggested the presence of subtle cerebral edema despite a lack of neurologic symptoms [7]. Additionally, long term memory deficits in children with type I diabetes mellitus (DM) are strongly associated with a past history of DKA, regardless of their overall glycemic control; this finding suggest that subtle neurological injury occurs during this critical time $[9,10]$.
The underlying mechanism for cerebral edema in children with DKA is unknown. Emerging evidence suggests that ischemic injury resulting from dehydration and hyperventilation due to acidosis may be an inciting factor [11-15]. Hypoperfusion and reperfusion injury with associated cerebral edema may develop as dehydration is correction and carbon dioxide levels normalize with treatment [6, 16-18]. Additionally, hyperglycemia and ketosis increase the risk of hyperemia during correction of carbon dioxide in animal studies [19]. Impaired cerebral autoregulation in other neurologic conditions such as stroke and traumatic brain injury is known to be associated with an increased risk of secondary injury and reperfusion injury [20, 21]. A transient impairment of cerebral autoregulation in DKA has been reported but overall data about changes in cerebral hemodynamics and autoregulation are limited in children with DKA [16, 22]. 
Understanding changes in cerebral hemodynamics during DKA and its impact upon neurologic injury may be useful to inform treatment strategies to optimize cerebral blood flow to minimize cerebral edema. Transcranial Doppler ultrasound (TCD) is a noninvasive test that can be used at the bedside to evaluate real time changes in cerebral hemodynamics and autoregulation. TCD is an established clinical tool in neurocritical care with applications that include monitoring for vasospasm after aneurysm subarachnoid hemorrhage, emboli detection during carotid endarterectomy, and screening for evidence of sickle cell vasculolopathy [23]. As a research tool, TCD has provided insight into cerebral blood flow patterns in a variety of neurological conditions such as acute ischemic stroke and traumatic brain injury.

We designed a study using TCD to evaluate blood flow patterns and changes in critically ill children with moderate to severe DKA. We hypothesized that decreased cerebral blood flow velocity and impaired autoregulation occurs early in DKA.

\section{Materials and Methods}

We performed a prospective observational study at a pediatric tertiary care center between 2011-2014. This study was approved by our institutional review board. Patients $\leq 18$ years old with moderate to severe DKA admitted to our pediatric intensive care unit (ICU) were screened. Patients were eligible for the study if they had a serum glucose $>200 \mathrm{mg} / \mathrm{dl}$, venous or capillary $\mathrm{pH}<7.15$, bicarbonate level $<15 \mathrm{mmol} / \mathrm{L}$, and ketones in their urine. We excluded patients with previously diagnosed diseases known to alter cerebral hemodynamics (e.g. Sickle cell disease). Informed consent was obtained from the patient's parent or guardian and assent was obtained from the patient when applicable.

Within 4 hours of admission to the intensive care unit, TCD was performed at the bedside using a $2-\mathrm{MHz}$ pulsed probe and commercially available TCD ultrasonography unit (Sonara Digital TCD, Carefusion, Middleton, WI) to measure the systolic, diastolic and mean flow velocities of the middle cerebral arteries (MCA) and basilar artery (BA). Arteries were insonated at 1millimeter ( $\mathrm{mm})$ intervals using previously described methods [24, 25]. Cerebral autoregulation was evaluated using the transient hyperemic response ratio (THRR) bilaterally, as previously described [26-28]. A THRR $<1.1$ was considered abnormal. Measurements were repeated after resolution of DKA which was defined as $\mathrm{pH}>7.3$, blood gas carbon dioxide $>35 \mathrm{mmHg}$, bicarbonate $>15 \mathrm{mmol} / \mathrm{L}$ or normalized anion gap and transition off of the insulin infusion to intermittent insulin with a regular diet.

Baseline characteristics of the patients including age, Glasgow Coma Scale (GCS), blood pH, blood glucose, bicarbonate, carbon dioxide, and neuroimaging results were recorded at the time of each study.

Treatment strategy, including choice and rate of fluids and imaging, was left to the discretion of the clinical team. The general practice within our institution utilizes an initial 10 milliliter per kilogram bolus of 0.9 normal saline followed by a two bag system with 0.9 normal saline based intravenous fluids at 1.5 to 2 times maintenance flow rate and an insulin drip of 0.1 units per kilogram per hour. The fluids are titrated to decrease blood glucose by approximately $100 \mathrm{mg} /$ $\mathrm{dl}$ per hour.

Statistical analysis was performed using GraphPad Prism ${ }^{\odot}$ (La Jolla, California). Descriptive statistics were calculated for baseline characteristics. Wilcoxon matched pairs test was used for comparisons. Each patient served as their own control and blood flow velocities obtained during DKA were compared with their values after resolution of DKA and also to previously published age and gender matched normal values. Baseline demographics and blood flow velocities for patients with cerebral edema versus those without cerebral edema were compared using non-parametric t-tests.

\section{Results}

\section{Baseline Characteristics}

26 total patients were enrolled. 15 patients were female (57\%). 17 patients (65\%) had newly diagnosed diabetes. Baseline characteristics are displayed in Table 1 . The median time from presentation to the emergency room to initial TCD study was 4.5 hours (2-6.5). Only the first study was obtained for 2 patients as they were discharged before the second TCD study was performed. Information for the first study for both of these patients was included in the data analysis.

Table 1: Baseline Patient Characteristics

\begin{tabular}{|l|c|c|}
\hline & Median & Range \\
\hline Age (Years) & 10 & $0.75-18$ \\
\hline Initial glucose (milligram/deciliter) & 558 & $289-1018$ \\
\hline Initial pH & 6.98 & $6.78-7.13$ \\
\hline Initial carbon dioxide (milliequivalent/liter) & 17 & $7-28$ \\
\hline $\begin{array}{l}\text { Initial bicarbonate } \\
\text { (milliequivalent/liter) }\end{array}$ & $<5$ & $<5-8$ \\
\hline Initial serum osmolality (calculated) & 313 & $278-368$ \\
\hline Sodium at time of 1st study & 144 & $134-157$ \\
\hline Sodium at time of 2nd study & 142 & $136-166$ \\
\hline Initial Glasgow Coma Scale (GCS) & 14 & $9-15$ \\
\hline
\end{tabular}

\section{Middle cerebral artery flow velocities}

No difference was found for systolic, diastolic or mean blood flow velocities for either middle cerebral artery during DKA compared to previously published age matched normal values (Figure 1). Additionally, there was no significant difference between the patients' systolic, diastolic or mean blood flow velocities for the right or left middle cerebral artery when comparing flow during the acute phase of DKA to flow after resolution of DKA in the same patients.

\section{Basilar artery flow velocities}

Basilar artery mean flow velocity was significantly lower during DKA than expected (median $47 \%$ of normal, range 33-129\%, $\mathrm{p}=$ 0.001 ) and remained significantly lower than normal after resolution of DKA $(\mathrm{p}=0.001)$ (Figure 2). 


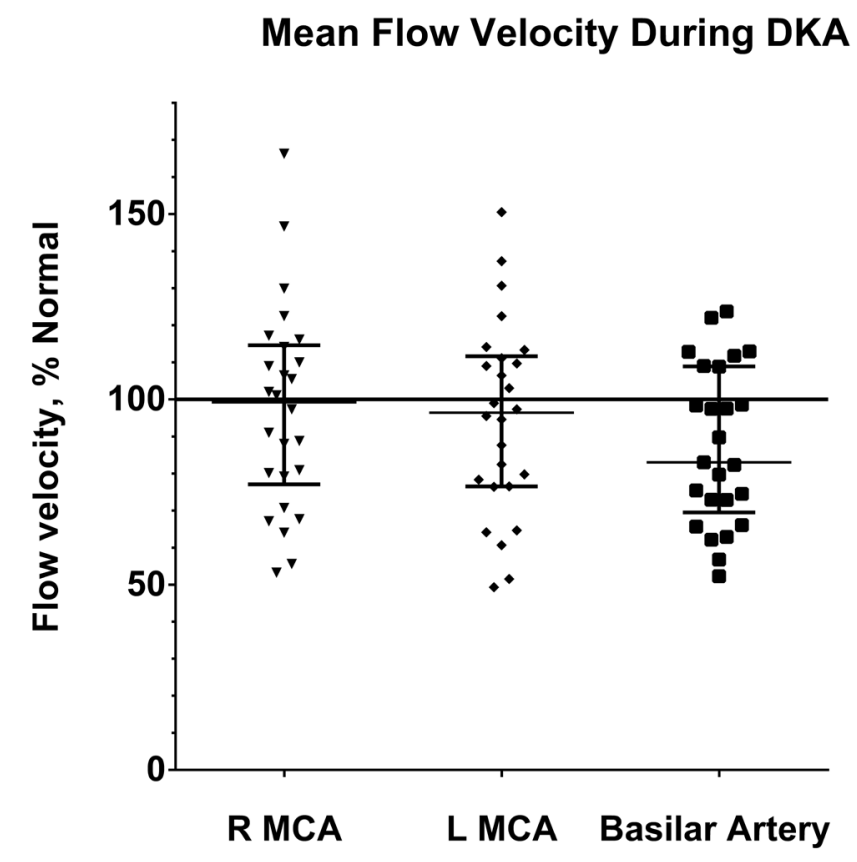

Figure 1. Mean flow velocities for intracranial blood vessels during diabetic ketoacidosis: Mean flow velocities are shown here as percentage of the expected value for the patient's age and gender. Median and interquartile ranges are displayed on the figure. No difference was found in the mean flow velocities of the right and left middle cerebral arteries during diabetic ketoacidosis compared to previously published age and gender matched normal values. Basilar artery mean flow velocity was significantly lower during DKA than expected $(\mathrm{p}=0.001)$.

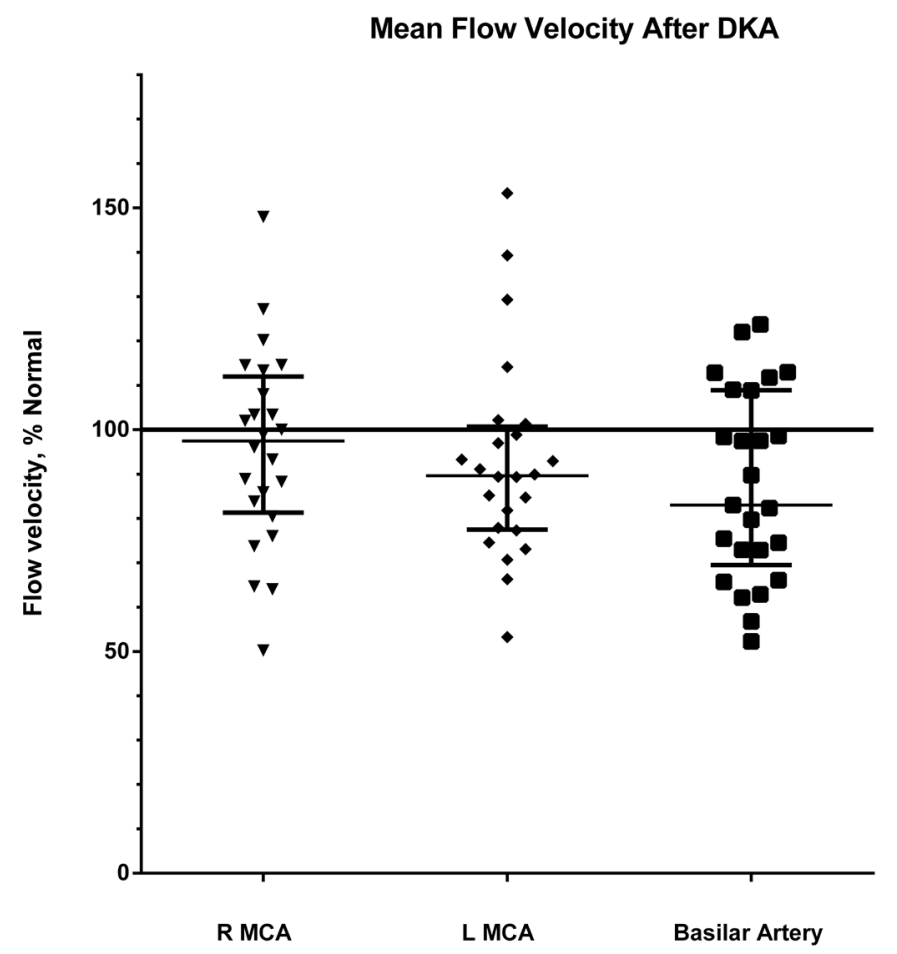

Figure 2. Mean flow velocities for intracranial blood vessels after resolution of diabetic ketoacidosis: Mean flow velocities are shown here as percentage of the expected value for the patient's age and gender. Median and interquartile ranges are displayed on the figure. No difference was found in the mean flow velocities of the right and left middle cerebral arteries during diabetic ketoacidosis compared to previously published age matched normal values. Basilar artery mean flow velocity was significantly lower after resolution of DKA than expected $(p=0.001)$. 


\section{MCA Autoregulation}
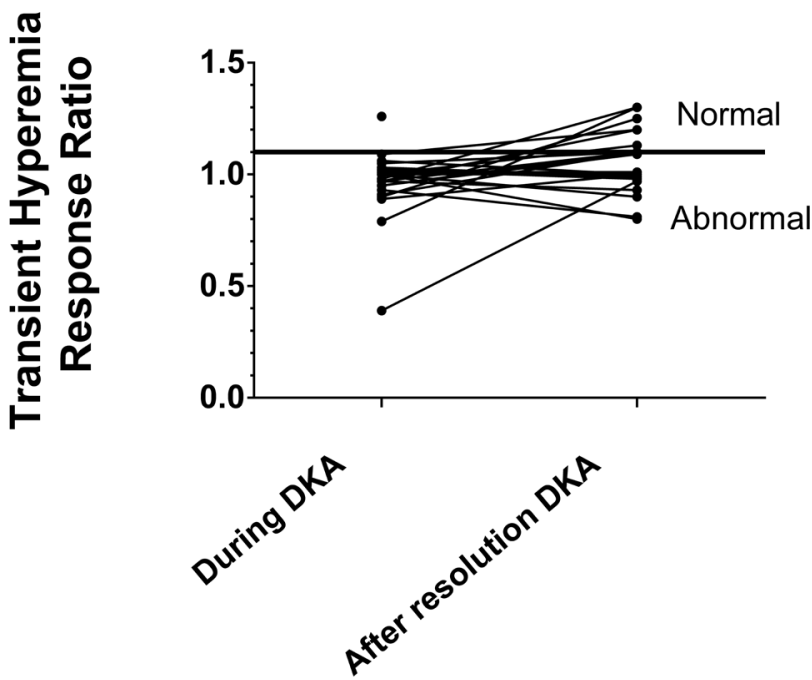

\section{R MCA Autoregulation}

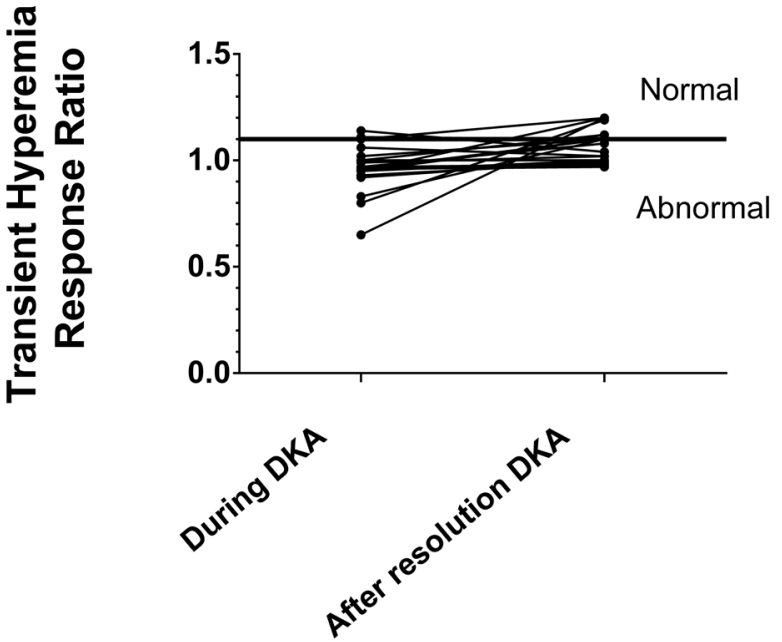

Figure 3a and b. Cerebral autoregulation for the middle cerebral arteries: Cerebral autoregulation was impaired during diabetic ketoacidosis in $92 \%$ patients as measured by the transient hyperemic response. A response of $\geq 1.1$ is defined as normal. a) The median transient hyperemic response on the right was $0.97(0.65-1.14)$ and b) on the left was $0.98(0.39-1.26)$. Followup testing was done in all but 2 patients after resolution of DKA and cerebral autoregulation remained abnormal in $64 \%$ of the patients.

\section{Cerebral autoregulation/THRR}

THRR data was obtained in 24 of the 26 patients; 2 patients did not tolerate testing due to agitation. THRR was abnormal, consistent with impaired cerebral autoregulation, in $92 \%$ of patients during DKA (Figure $3 \mathrm{a}$ and $\mathrm{b}$ ) and remained abnormal in $64 \%$ of patients on the second evaluation after DKA had resolved.

\section{Patients with abnormal imaging}

10 patients had head imaging on presentation. 6 patients had evidence of cerebral edema (CE) and 1 of these patients also had a left basal ganglia infarction. The mean basilar artery flow velocities were lower in patients with clinical and radiographic evidence of $\mathrm{CE}$ than patients without $\mathrm{CE}(\mathrm{p}=0.06)$. No difference was seen in the middle cerebral artery flow velocities in patients with and without CT abnormalities. Initial GCS was significantly lower in patients with CE (median 10) than patients without edema [14], $\mathrm{p}=0.01$. There was no difference in age, initial $\mathrm{pH}$, glucose, bicarbonate or $\mathrm{CO} 2$ between patients with and without CE.

\section{Discussion}

DKA resulting in cerebral edema is a leading cause of immediate morbidity and mortality in children with diabetes mellitus. Episodes of DKA may contribute to long-term morbidity and cognitive impairment in these children with diabetes mellitus as well $[2-4,9$, 10]. Changes that occur in the brain that lead to cerebral edema and long-term neurological injury after DKA are poorly understood. In order to develop strategies to minimize and prevent the neurologic sequelae of DKA, we must first understand the alterations in cerebral blood flow that occur during DKA and which changes are maladaptive.
This study provides further insight into the changes in cerebral blood flow and autoregulation in critically ill children with DKA that may contribute to intracranial complications.

No difference from expected age and gender matched normal values was found in the cerebral blood flow velocities of the left and right middle cerebral artery (MCA) during DKA. This is an interesting finding as these flow velocities were within the normal range for age and gender despite acidosis and hypocapnea, which should presumably result in decreased cerebral blood flow. This may represent an adaptive response of the child to try to maintain an adequate cerebral perfusion pressure. Prior studies including TCD studies by Roberts et al reported hyperemia as early as 2 hours into treatment for DKA $[16,29,30]$. The difference in our study results versus the Roberts et al. study may be related to what was considered a normal cerebral blood flow velocity for the patient. We compared the cerebral blood flow velocities to previously published age and gender matched normal values [24, 25]. The authors of the Roberts et al. study compared the measured blood flow velocities to values that were corrected for the patient's carbon dioxide level and reported a relative hyperemia for a given carbon dioxide level; patients in this study were assumed to have intact $\mathrm{CO} 2$ reactivity, which may not have been true. We also compared each patient's cerebral flow velocities during DKA to their flow velocities after resolution DKA and found no difference between values during and after DKA. This use of each patient as their own control also suggests that patients were not significantly hyperemic during DKA. The patients with cerebral edema on neuroimaging were not any more likely to have hyperemia on their TCDs than patients without clinical or imaging evidence of cerebral edema. 
Unlike the MCAs, the mean flow velocities for the basilar artery were significantly lower than expected both during and after resolution of DKA; this study is the first to report changes in the BA flow velocity in children with DKA. These lower blood flow velocities may represent a relative failure of the posterior circulation to increase cerebral blood flow to maintain a constant cerebral perfusion pressure in a hypocarbic state. These diminished flows may result in ischemia during DKA. Glaser et al. reported that in magnetic resonance imaging of children during DKA, the occipital lobes had lower apparent diffusion coefficient values during DKA than other areas of the brain and those values continued to be lower during treatment [31]. Low apparent diffusion coefficient values suggest cytotoxic edema. Furthermore, children with cerebral edema also had significantly lower basilar flow velocities than patients with DKA but without cerebral edema in this study, which suggests that these abnormalities in cerebral flow may be a key feature in the development of intracranial injury during DKA. Notably, the hippocampus, which is important in cognition and formation of memories, is supplied by the posterior circulation; thus it is possible that hypoperfusion to the hippocampus contributes to the long term cognitive and memory deficits in patients with a history of DKA.

Our findings in combination of those of Glaser et al. suggest that the posterior circulation and occipital lobe may have a unique vulnerability to initial and prolonged cerebral ischemia during DKA in children. It is unclear why only the posterior circulation showed a difference in our study but this result may be due to limited power from the small sample size. Alternatively, glucose dysregulation may preferentially affect the posterior circulation involvement since hypoglycemia is known to predominantly cause parietal-occipital lobe ischemia $[32,33]$. A recent study of children with type I diabetes also found that $>50 \%$ of them had focal slowing in the bilateral posterior region on electrocephalogram at the time of diagnosis; this finding also suggests sensitivity of the posterior brain to injury with abnormalities in glucose homeostasis [34].

Cerebral autoregulation was impaired in almost all patients in this study and remained abnormal in the majority of patients after correction of lab abnormalities. In other studies, autoregulation has been found to be abnormal in patients with type II diabetes and possibly longstanding type I diabetes presumably due to chronic endothelial injury and inflammation [16]. However, this is not the most likely explanation for impaired autoregulation in this study as the majority of the patients were newly diagnosed with diabetes. Abnormal autoregulation as seen in this study is more likely an acute change related to DKA. The reasons for this are not completely clear but one explanation may be that this prolonged loss of cerebral autoregulation is due to the fact that the cerebrospinal buffering system normalizes slowly in comparison to the faster normalization of the serum bicarbonate and $\mathrm{pH}$. Two prior studies have looked at cerebral autoregulation during DKA in children. The first study reported that autoregulation, based upon the autoregulatory index, was abnormal in 5 of 6 patients during DKA but that cerebral autoregulation normalized by 30 hours from presentation [16]. A follow-up study by this group reported impairment in $40 \%$ of the patients with DKA and normalization of cerebral autoregulation in the majority of patients by 36-72 hours of therapy [22]. In contrast, our study found that a prolonged abnormality in cerebral autoregulation in the majority of our patients persisted even after labs had normalized. The difference in our results from those previously published by Ma et. al. may be related to how autoregulation was evaluated. In the Ma et al study, the method used to evaluate autoregulation utilized changes in the position of the head of the bed; however, with this approach, measurements can be confounded by the fact that intracranial pressure also may be influenced by bed position. The technique utilized in our study with transient compression of the internal carotid artery does not directly influence ICP.

Under normal conditions, cerebral autoregulation allows the brain to maintain a steady cerebral blood flow despite changes in blood pressure. With loss of normal autoregulation, patients are at risk for cerebral ischemia or hyperemia with small changes in blood pressure. A recent study by Deeter at al. reported of children with DKA found that 19 of 33 patients had hypertension before treatment and $82 \%$ had hypertension within the first 6 hours of admission [35]. About a quarter of the patients had continued hypertension after discharge. This observation of increased blood pressure may represent a physiologic response of the body during DKA to try to maintain cerebral blood flow in opposition to cerebral vasoconstriction due to hypocapnea. However, if patients have vasomotor paralysis as seen in our study, then this adaptive hypertension may cause harm by resulting in hyperemia and cerebral edema. These results suggest that patients may benefit from prolonged neuroprotective strategies such as tight blood pressure control because of persistent impairment in cerebral autoregulation even after lab values normalize.

These prolonged abnormalities in cerebral blood flow in the posterior circulation and cerebral autoregulation may contribute to the subtle cerebral injury and thus the longstanding cognitive and behavioral deficits that have been reported in children with DKA [36-38]. A single episode of DKA has been shown to been associated with cognitive dysfunction in rats [39]. There also is evidence of permanent cerebral injury after DKA in children. Patients with a single episode DKA have evidence of decreased gray matter and decreased $\mathrm{N}$-acetylaspartate/creatine ratios on imaging [38, 40]. Thus, these acute derangements in cerebral hemodynamics even with a single episode of DKA may have long-term implications for the children's neurologic outcome.

This pilot study is limited primarily by the small sample size and confirmation of our findings in larger studies of children with DKA need to be performed. Future studies should evaluate whether there is a correlation in neuropsychological measures with decreased basilar flow in patients. Also, future studies should evaluate children days and weeks out from presentation of DKA to determine how long alterations in cerebral autoregulation persist after resolution of DKA.

\section{Conclusion}

Basilar artery blood flow was decreased during DKA in our study and remained low after resolution of DKA. This may contribute to ischemic injury in these patients. Our data also suggests prolonged impairment of cerebral autoregulation occurs in children during DKA and persists after correction of lab abnormalities. Future larger 
studies are needed to further characterize the timing and changes to cerebral hemodynamics during DKA and any association with acute and long term neurological consequences for children with type 1 DM. Understanding these disruptions in cerebral blood flow may lead to future therapeutic targets with the goal to improve morbidity and cognitive outcomes of these children.

\section{Financial support for study: none}

\section{References}

1. Bello FA, Sotos JF (1990) Cerebral oedema in diabetic ketoacidosis in children. Lancet 336: 64. [crossref]

2. $\quad$ Edge JA, Jakes RW, Roy Y, et al. (2006) The UK case-control study of cerebral oedema complicating diabetic ketoacidosis in children. Diabetologia 49: 2002-2009

3. Lawrence SE, Cummings EA, Gaboury I, Daneman D (2005) Population-based study of incidence and risk factors for cerebral edema in pediatric diabetic ketoacidosis. J Pediatr 146: 688-692. [crossref]

4. Hanas R, Lindgren F, Lindblad B (2007) Diabetic ketoacidosis and cerebral oedema in Sweden--a 2-year paediatric population study. Diabet Med 24: 1080-1085. [crossref]

5. Edge JA, Hawkins MM, Winter DL, Dunger DB (2001) The risk and outcome of cerebral oedema developing during diabetic ketoacidosis. Arch Dis Child 85: 16-22. [crossref]

6. Figueroa RE, Hoffman WH, Momin Z, et al. (2005) Study of subclinical cerebral edema in diabetic ketoacidosis by magnetic resonance imaging $\mathrm{T} 2$ relaxometry and apparent diffusion coefficient maps. Endocr Res 31:345-355

7. Glaser NS, Wootton-Gorges SL, Buonocore MH, Marcin JP, Rewers A, et al. (2006) Frequency of sub-clinical cerebral edema in children with diabetic ketoacidosis. Pediatr Diabetes 7: 75-80. [crossref]

8. Muir AB, Quisling RG, Yang MC, et al. (2004) Cerebral edema in childhood diabetic ketoacidosis: natural history, radiographic findings, and early identification. Diabetes Care 27:1541-1546

9. Krane EJ, Rockoff MA, Wallman JK, Wolfsdorf JI (1985) Subclinical brain swelling in children during treatment of diabetic ketoacidosis. $N$ Engl J Med 312: 1147-1151. [crossref]

10. Ghetti S, Lee JK, Sims CE, Demaster DM, Glaser NS (2010) Diabetic ketoacidosis and memory dysfunction in children with type 1 diabetes. J Pediatr 156: 109-114. [crossref]

11. Yuen N, Anderson SE, Glaser N, Tancredi DJ, O'Donnell ME (2008) Cerebral blood flow and cerebral edema in rats with diabetic ketoacidosis. Diabetes 57: 2588-2594. [crossref]

12. Wootton-Gorges SL, Buonocore MH, Kuppermann N, et al. (2007) Cerebral proton magnetic resonance spectroscopy in children with diabetic ketoacidosis. AJNR Am J Neuroradiol 28: 895-899

13. Salem MA, Matta LF, Tantawy AA, et al. (2002) Single photon emission tomography (SPECT) study of regional cerebral blood flow in normoalbuminuric children and adolescents with type 1 diabetes. Pediatr Diabetes 3: 155-162

14. Foster JR, Morrison G, Fraser DD (2011) Diabetic ketoacidosis-associated stroke in children and youth. Stroke Res Treat 2011: 219706. [crossref]

15. Duckrow RB (1995) Decreased cerebral blood flow during acute hyperglycemia Brain Res 703: 145-150. [crossref]

16. Vavilala MS, Souter MJ, Lam AM (2005) Hyperemia and impaired cerebral autoregulation in a surgical patient with diabetic ketoacidosis. Can J Anaesth 52: 323-326. [crossref]

17. Vavilala MS, Richards TL, Roberts JS, et al. (2010) Change in blood-brain barrier permeability during pediatric diabetic ketoacidosis treatment. Pediatr Crit Care Med 11:332-338

18. Vavilala MS, Marro KI, Richards TL, et al. (2011) Change in mean transit time, apparent diffusion coefficient, and cerebral blood volume during pediatric diabetic ketoacidosis treatment. Pediatr Crit Care Med 12: e344-349

19. Glaser N, Bundros A, Anderson S, Tancredi D, Lo W, et al. (2014) Brain cell swelling during hypocapnia increases with hyperglycemia or ketosis. Pediatr Diabetes 15: 484-493. [crossref]

20. Jaeger M, Schuhmann MU, Soehle M, et al. (2006) Continuous assessment of cerebrovascular autoregulation after traumatic brain injury using brain tissue oxygen pressure reactivity. Crit Care Med 34:1783-1788

21. Lang EW, Czosnyka M, Mehdorn HM (2003) Tissue oxygen reactivity and cerebral autoregulation after severe traumatic brain injury. Crit Care Med 31: 267-271. [crossref]

22. Ma L, Roberts JS, Pihoker C, et al. (2014) Transcranial Doppler-based assessment of cerebral autoregulation in critically ill children during diabetic ketoacidosis treatment. Pediatr Crit Care Med 15:742-749
23. Sloan MA, Alexandrov AV, Tegeler CH, et al. (2004) Assessment: transcranial Doppler ultrasonography: report of the Therapeutics and Technology Assessment Subcommittee of the American Academy of Neurology. Neurology 62:1468-1481

24. Bode H, Eden A (1989) Transcranial Doppler sonography in children. J Child Neurol 4 Suppl: S68-76. [crossref]

25. Bode H, Wais U (1988) Age dependence of flow velocities in basal cerebral arteries. Arch Dis Child 63: 606-611. [crossref]

26. Aaslid R, Newell DW, Stooss R, et al. (1991) Assessment of cerebral autoregulation dynamics from simultaneous arterial and venous transcranial Doppler recordings in humans. Stroke 22:1148-1154

27. Smielewski P, Czosnyka M, Kirkpatrick P, McEroy H, Rutkowska H, et al. (1996) Assessment of cerebral autoregulation using carotid artery compression. Stroke 27: 2197-2203. [crossref]

28. Giller CA (1991) A bedside test for cerebral autoregulation using transcranial Doppler ultrasound. Acta Neurochir 108:7-14

29. Glaser NS, Tancredi DJ, Marcin JP, et al. (2013) Cerebral hyperemia measured with near infrared spectroscopy during treatment of diabetic ketoacidosis in children. $J$ Pediatr 163:1111-1116

30. Roberts JS, Vavilala MS, Schenkman KA, et al. (2006) Cerebral hyperemia and impaired cerebral autoregulation associated with diabetic ketoacidosis in critically ill children. Crit Care Med 34:2217-2223

31. Glaser NS, Wootton-Gorges SL, Marcin JP, Buonocore MH, Dicarlo J, et al. (2004) Mechanism of cerebral edema in children with diabetic ketoacidosis. J Pediatr 145 : 164-171. [crossref]

32. Tam EW, Widjaja E, Blaser SI, et al. (2008) Occipital lobe injury and cortical visual outcomes after neonatal hypoglycemia. Pediatrics 122: 507-512

33. Vijay K, Agarwal A (2010) Neonatal hypoglycemia resulting in occipital cerebral injury. Pediatr Radiol 40 Suppl 1: S178. [crossref]

34. Mackay MT, Molesworth C, Northam EA, et al. (2015) Diabetic ketoacidosis and electroencephalographic changes in newly diagnosed pediatric patients. Pediatr Diabetes 17: 244-248

35. Deeter KH, Roberts JS, Bradford H, et al. (2011) Hypertension despite dehydration during severe pediatric diabetic ketoacidosis. Pediatr Diabetes 12: 295-301

36. Nadebaum C, Scratch SE, Northam EA, et al. (2012) Clinical utility of mental state screening as a predictor of intellectual outcomes 6 months after diagnosis of type 1 diabetes. Pediatr Diabetes 13: 632-637

37. Northam EA, Anderson PJ, Jacobs R, Hughes M, Warne GL, et al. (2001) Neuropsychological profiles of children with type 1 diabetes 6 years after disease onset. Diabetes Care 24: 1541-1546. [crossref]

38. Cameron FJ, Scratch SE, Nadebaum C, et al. (2014) Neurological consequences of diabetic ketoacidosis at initial presentation of type 1 diabetes in a prospective cohort study of children. Diabetes Care 37: 1554-1562

39. Glaser N, Anderson S, Leong W, Tancredi D, O'Donnell M (2012) Cognitive dysfunction associated with diabetic ketoacidosis in rats. Neurosci Lett 510: 110-114. [crossref]

40. Wootton-Gorges SL, Buonocore MH, Caltagirone RA, et al. (2010) Progressive decrease in $\mathrm{N}$-acetylaspartate/Creatine ratio in a teenager with type 1 diabetes and repeated episodes of ketoacidosis without clinically apparent cerebral edema: Evidence for permanent brain injury. AJNR 31:780-781

\section{Citation:}

Melissa G Chung, Tensing Maa and Nicole F O'Brien (2017) Changes in Cerebral Blood Flow and Prolonged Disturbances in Cerebral Autoregulation in Critically ill Children with Diabetic Ketoacidosis. Endocrinol Diabetes Metab $J$ Volume 1(1): 1-6 